\title{
Quantitative evaluation of fundus autofluorescence imaged "in vivo" in eyes with retinal disease
}

\author{
Noemi Lois, Anthony S Halfyard, Alan C Bird, Frederick W Fitzke
}

\begin{abstract}
Aim-To describe a new method of evaluating the topographic distribution of fundus autofluorescence in eyes with retinal disease.

Methods-Images of fundus autofluorescence were obtained in five patients and 34 normal volunteers using a confocal scanning laser ophthalmoscope (cSLO). To evaluate the topographic distribution of fundus autofluorescence throughout the posterior pole a rectangular box, $10 \times 750$ pixels, was used as the area of analysis. The box was placed, horizontally, across the macular region. The intensity of fundus autofluorescence of each pixel within the rectangular box was plotted against its degree of eccentricity. Profiles of fundus autofluorescence from patients were compared with those obtained from the age matched control group and with cSLO images.
\end{abstract}

Results-Profiles of fundus autofluorescence appeared to represent the topographic distribution of fundus autofluorescence throughout the posterior pole appreciated in the cSLO images, and allowed rapid identification and quantification of areas of increased or decreased fundus autofluorescence.

Conclusions-Fundus autofluorescence profiles appear to be useful to study the spatial distribution of fundus autofluorescence in eyes with retinal disease.

(Br F Ophthalmol 2000;84:741-745)

Science, Institute of

Ophthalmology,

London

$\mathrm{N}$ Lois

A S Halfyard

A C Bird

F W Fitzke

Medical Retina

Service, Moorfields

Eye Hospital, London

$\mathrm{N}$ Lois

A C Bird

Vitreo-Retinal Service, St Paul's Eye Unit, The

Royal Liverpool

University Hospital,

Liverpool

N Lois

Correspondence to: Professor Frederick W Fitzke, Institute of Ophthalmology, London f.fitzke@ucl.ac.uk

Accepted for publication 24 February 2000 information regarding the distribution of fundus autofluorescence may provide insight on
RPE cells and photoreceptors. ${ }^{17} 19$ Hence, the sequence of events that lead to retina damage in a variety of retinal diseases. Until recently, information regarding the content and spatial distribution of RPE lipofuscin in eyes with retinal disease was limited by the scarce number of eyes available for histopathological evaluation. This problem has been recently overcome with the development of new techniques that allow the study of RPE lipofuscin levels "in vivo". ${ }^{20-25}$ Using a spectrophotometer ${ }^{20-22}$ and a confocal scanning laser ophthalmoscope (cSLO) $)^{23-25}$ the level of fundus autofluorescence (index of RPE lipofuscin content) has been assessed "in vivo" in a variety of retinal disorders. Previous in vivo studies have quantified fundus autofluorescence in eyes with retinal disease at selected areas of the retina. ${ }^{20-25}$ These isolated measurements have provided valuable information in a number of retinal disorders. However, the differential distribution of fundus autofluorescence, appreciated in the images obtained using a cSLO,${ }^{23-25}$ can not be represented by an isolated measurement.

The aim of the present study was to design a method of representing the topographic distribution of fundus autofluorescence in eyes with retinal disease. In order to test this technique, fundus autofluorescent studies were performed in a group of patients with inherited retinal disorders and in an age matched control group.

\section{Subjects and methods}

Five patients with retinal disease and 34 normal volunteers were included in the study. The median age of patients was 29 years (range 20-36 years), and of normal volunteers 30 years (range 20-36 years). One patient with each of Best disease, adult vitelliform foveomacular dystrophy, Stargardt macular dystrophy-fundus flavimaculatus, bull's eye macular dystrophy, and cone-rod dystrophy were included in the study. Fundus examination of normal volunteers disclosed no abnormalities. All subjects had clear lenses on slit lamp biomicroscopy.

In all subjects one eye was selected and levels of fundus autofluorescence were recorded. Images of fundus autofluorescence were obtained, after pupillary dilatation, using a confocal scanning laser ophthalmoscope (cSLO, Zeiss, Oberkochen, Germany), as has been previously described. ${ }^{26}$ Briefly, autofluorescent images were produced using a 40 degree field of view mode, and a confocal aperture which provided a full width half maximum depth resolution of less than $600 \mu \mathrm{m}$. The ametropic 
corrector was employed to correct for refractive error and to focus on the structure of interest. An argon laser light (488 $\mathrm{nm})$ was used for illumination, and a wide band pass filter with short wavelength cut off $(521 \mathrm{~nm})$ was inserted in front of the detector to record fundus autofluorescence. The argon laser was measured at regular intervals and found to have a constant power output of $250 \mu \mathrm{W}$ at the cornea. The automatic gain control was switched off and the instrument gain was kept constant for all measurements of normal volunteers and patients. A series of images of the eye studied was recorded at standard video scanning rates on VHS video tape. Later, 32 consecutive frames were digitised, aligned, and averaged to obtain each image. Since all normal volunteers and patients had clear lenses, no correction for lens opacities was made

In order to analyse the topographic distribution of fundus autofluorescence the following computer program was designed and written. A rectangular box, 10 pixels in height by 750 pixels in width, was used as the area of analysis. The box was placed, horizontally, across the macular region with its centre located at the centre of the fovea. In patients in whom the centre of the fovea was difficult to recognise its location was estimated based on its expected anatomical location. The centre of the fovea was considered as 0 degrees of eccentricity.

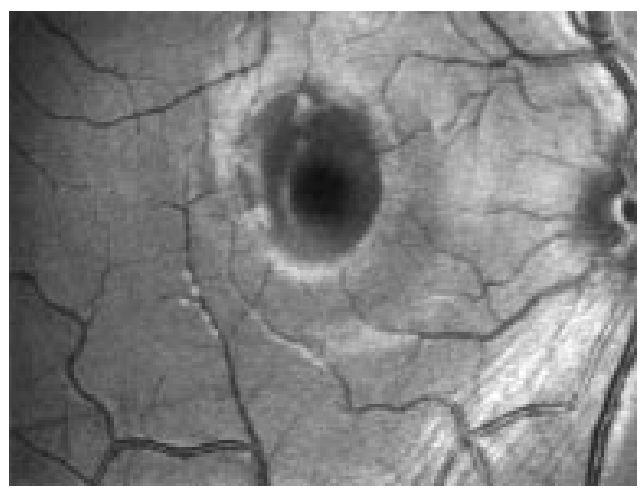

The length of the box allowed the study of fundus autofluorescence in an area extending from the optic nerve to the temporal aspect of the macula (12-16 degrees of eccentricity). For each subject the coordinates corresponding to the centre of the fovea, the horizontal line corresponding to the area of analysis, and the nasal and temporal ends of the area studied were marked and saved in the fundus autofluorescent image (Fig 1).

The intensity of fundus autofluorescence was measured in grey scale arbitrary units. The value of fundus autofluorescence of each pixel within the rectangular box was plotted against its degree of eccentricity with respect to the fovea, after having subtracted the video offset grey scale measured over the optic nerve head. Thus, the autofluorescence intensity of each of the 10 pixels within the width of the box at a particular degree of eccentricity was represented in a graph as an individual point depending on its value of autofluorescence. In patients, the highest, mean, and lowest values of each of the 10 pixels throughout the entire area studied were indicated in the graph as continuous lines (Fig 1).

A plot was generated with the $25 \%$ and $75 \%$ confidence interval (lower and upper quartiles) and the mean of the mean, lowest and highest values obtained at each degree of eccentricity for all normal volunteers. Single plots of fundus autofluorescence obtained from each
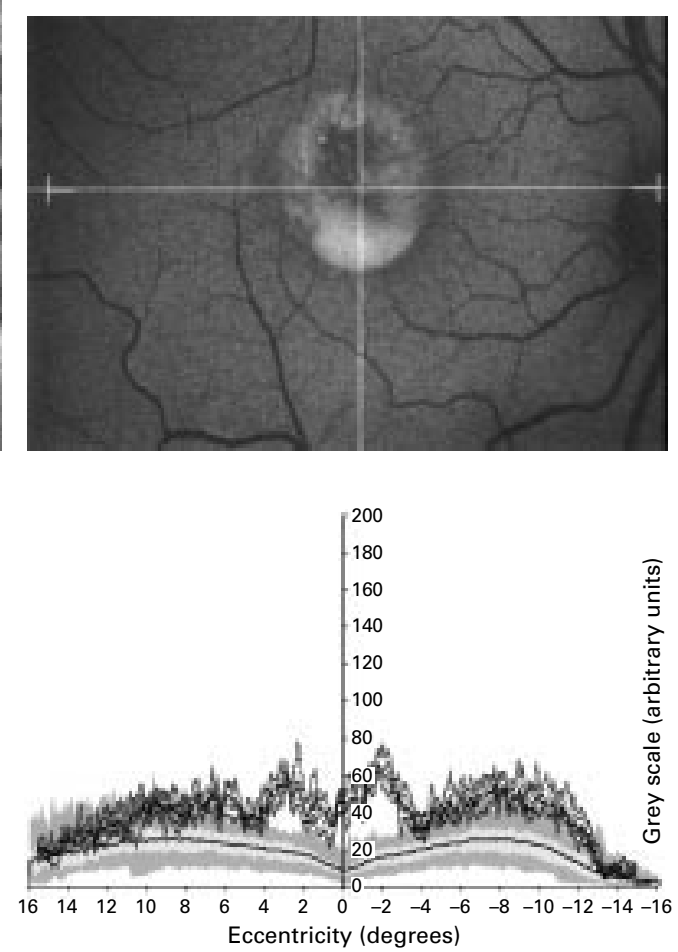

Figure 1 (Left top) Fundus photograph (red-free) of a 20 year old woman with Best's disease. (Right top) Fundus autofluorescent image of the same patient. The intersection between the horizontal and vertical lines represents the centre of the fovea. The horizontal line shows the area where measurements were taken. The right and left ends of the area of analysis are also marked on the image. (Right bottom) Fundus autofluorescent profile (dotted graph). Dark dots represent values of fundus autofluorescence of single pixels within the area studied. The highest, mean, and lowest values of fundus autofluorescence are represented by continuous dark lines (top, medium, and bottom lines, respectively). A graph with values of fundus autofluorescence corresponding to an age matched control group is also shown. Two light grey bands represent the $25 \%$ (inferior grey band) and 75\% (superior grey band) confident interval. The mean (black line), lowest (inferior aspect of the dark grey band), and highest (superior aspect of the dark grey band) values of fundus autofluorescence at each degree of eccentricity are also represented. The regional increased autofluorescence is localised to the centre of the macula. 

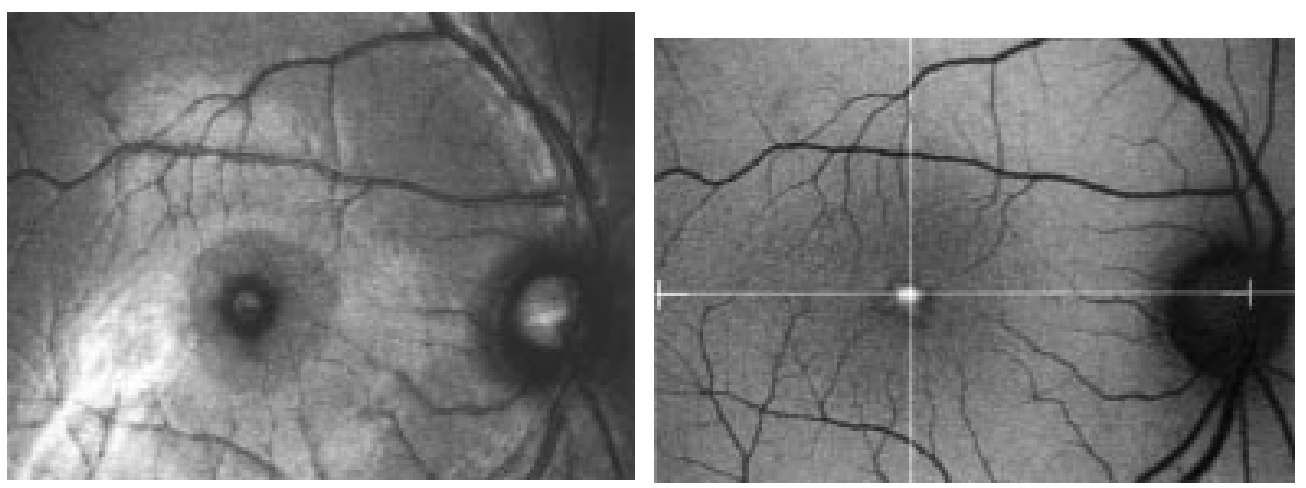

Figure 2 (Left top) Fundus photograph (red-free) of a 36 year old man with adult vitelliform foveomacular dystrophy. Fundus autofluorescent image (right top) and fundus autofluorescent profile of the same patient (right bottom). Very high levels of fundus autofluorescence were detected at the centre of the fovea. Background values of fundus autofluorescence were also high throughout the entire area studied.

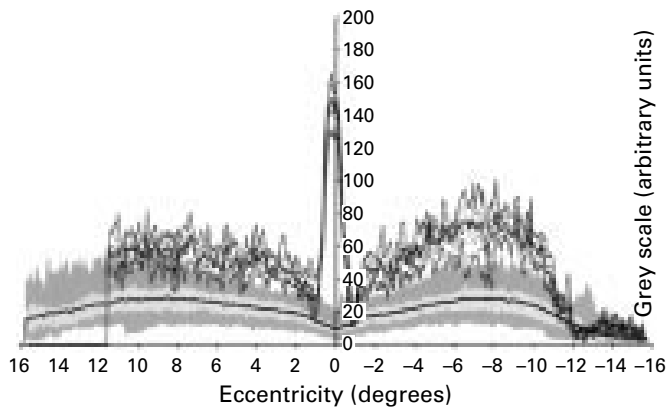

patient were compared with cSLO images and with the plot obtained from the age matched control group (Figs 1-5).

\section{Results}

In patients with retinal disease, topographic plots demonstrated well the spatial distribution of fundus autofluorescence throughout the
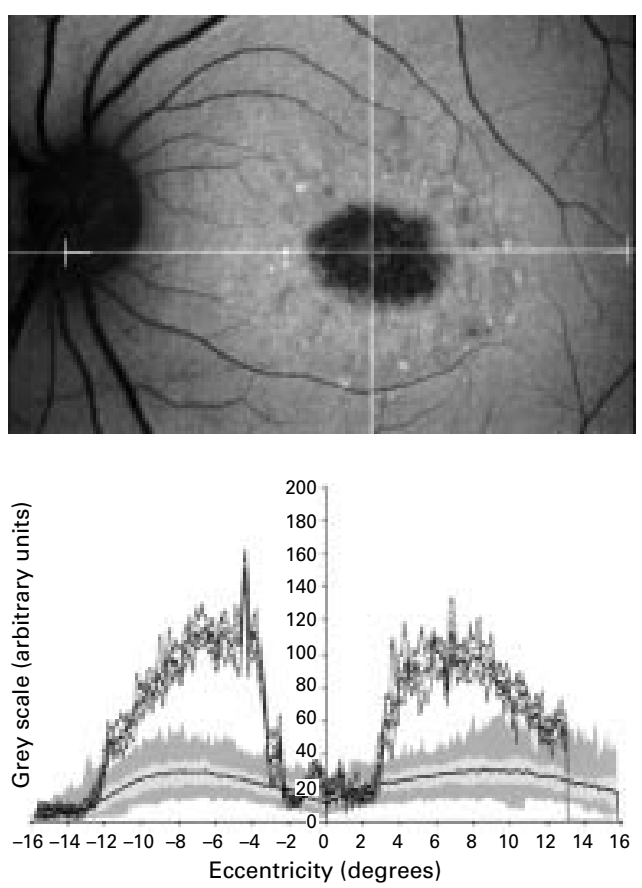

Figure 3 Fundus autofluorescent image of a 35 year old woman with Stargardt macular dystrophy-fundus flavimaculatus (top). An area of decreased signal at the centre of the macula and small foci of increased signal within it and around it were seen. Fundus autofluorescence profile showed very high background fundus autofluorescence levels across the entire area studied. Values of fundus autofluorescence at the fovea were within the lower normal values and peaks of increased autofluorescence at that site were also detected (bottom).

posterior pole seen in cSLO images (Figs 1-5). Thus, high levels of fundus autofluorescence detected at the centre of the macula in a patient with Best's disease corresponded well with the intense signal from the "pseudohypopyon" lesion observed in the cSLO image (Fig 1). Similarly, high levels of fundus autofluorescence were observed at the centre of the fovea in a patient with adult vitelliform foveomacular dystrophy corresponding with an area of intense signal in the cSLO image (Fig 2). In a patient with Stargardt macular dystrophy-
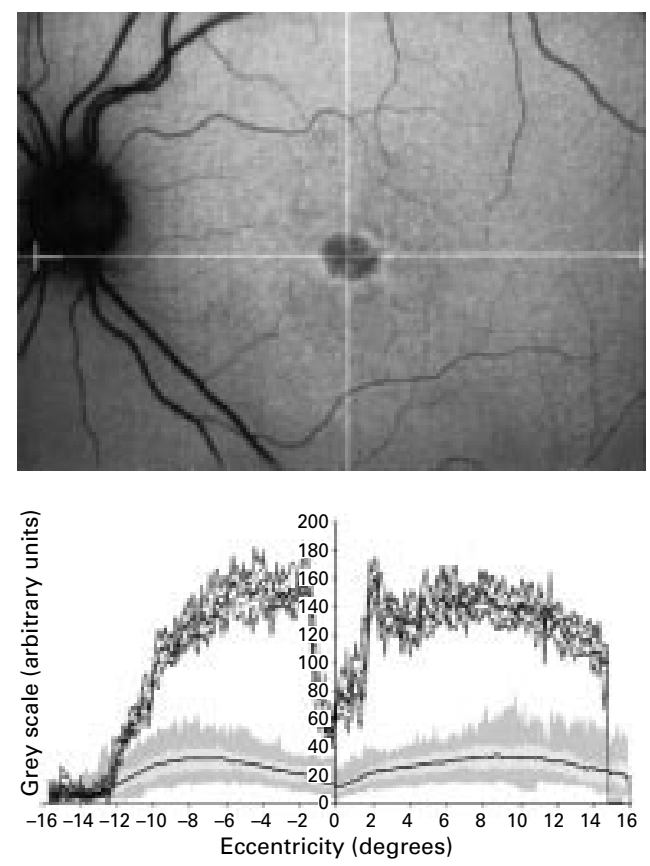

Figure 4 Fundus autofluorescent image of a 28 year old female with a bull's eye macular dystrophy (top). No focal areas with high or low intensity signal were detected outside the fovea in the cSLO image. Fundus autofluorescence profile demonstrated very high levels of fundus autofluorescence throughout the posterior pole (bottom). 

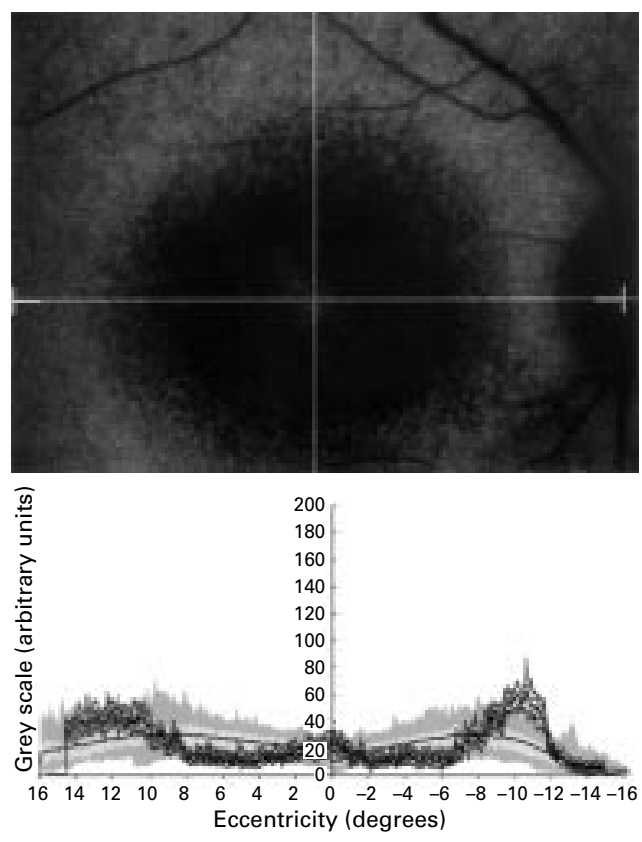

Figure 5 Fundus autofluorescence profile in a 32 years old man with cone-rod dystrophy. A large area with low intensity signal was observed at the macula in the cSLO image (top). This area appeared to be surrounded by a ring of high intensity signal. The fundus autofuorescence profile disclosed levels of fundus autofluorescence at the centre of the macula within the lower normal range and high levels of fundus autofluorescence outside the centre.

fundus flavimaculatus several peaks of increased fundus autofluorescence appeared to correspond well with foci of increased signal from "flecks" observed in the cSLO image (Fig 3). In a patient with bull's eye macular dystrophy no focal changes in fundus autofluorescence were detected outside the centre of the macula (Fig 4). In a patient with cone-rod dystrophy low levels of fundus autofluorescence were measured at the macula corresponding to a large area with low intensity signal in the cSLO image (Fig 5).

Topographic plots allowed also the study of background fundus autofluorescence, measured outside areas of focal increased or decreased fundus autofluorescence. Background values of fundus autofluorescence were found to be high throughout the posterior pole in patients with adult vitelliform foveomacular dystrophy, Stargardt macular dystrophy -fundus flavimaculatus, and bull's eye macular dystrophy (Figs 2-4).

\section{Discussion}

There appears to be a clear value in studying the distribution of fundus autofluorescence in eyes with retinal disorders. Hence, it may provide a greater understanding of the pathogenetic mechanisms of retinal disease. Similarly, it may explain the preferential regional involvement observed in certain retinal disorders, such as the perifoveal involvement that occurs in early stages of age related macular degeneration. In addition, the distribution of fundus autofluorescence may be helpful to predict the extension of further retinal damage - that is, high levels of fundus autofluorescence involving the posterior pole may indicate the development of a large area of atrophy whereas a small restricted area of increased autofluorescence may suggest a more favourable outcome.

In previous reports, Delori and associates measured fundus autofluorescence at $7 \mathrm{de}-$ grees temporal to the fovea and at the fovea in eyes with retinal disease, where the highest and lowest levels of fundus autofluorescence have been found in normal volunteers. ${ }^{20-22}$ Von Ruckmann and colleagues used as the area of analysis an $8 \times 8$ pixel box located anywhere in the fundus where no focal changes were observed to obtain background levels of autofluorescence. ${ }^{24}{ }^{25}$ Although studying levels of fundus autofluorescence at 7 degrees temporal to the fovea and at the fovea provides valuable information and allows comparison between individuals, it remains unclear if those locations are the most appropriate to evaluate fundus autofluorescence in all retinal diseases. Similarly, measuring fundus autofluorescence in a selected area of the fundus has several limitations. Firstly, it may not allow accurate comparisons between patients, since the eccentricity selected to measure fundus autofluorescence may be different in different individuals. Secondly, if it is proposed to study changes in fundus autofluorescence over time, autofluorescence measurements should be obtained always at the same area for all follow up visits, which may be difficult if this approach is used. Lastly, the differential distribution of fundus autofluorescence throughout the fundus, appreciated in the images obtained using a cSLO $^{23-25}$ can not be represented by an isolated measurement.

In this study, topographic plots were used to represent the differential distribution of fundus autofluorescence in eyes with retinal disease. This method of analysis allowed measurement of fundus autofluorescence throughout a large area of the posterior pole and provided a good estimate of variations in fundus autofluorescence across this region. Quantitative values of fundus autofluorescence could be compared with the qualitative findings observed in the cSLO images. Similarly, fundus autofluorescent profiles obtained from patients could be compared with those obtained from an age matched control group, allowing an estimation of areas of increased/decreased fundus autofluorescence. In addition, since the software used records the coordinates corresponding to the area of analysis, it would be possible to evaluate changes of fundus autofluorescence in the area studied over time.

The purpose of plotting values of fundus autofluorescence of single pixels throughout the entire area studied instead of the average value of a certain number of pixels, was to avoid the smoothing effect in the values of autofluorescence that would occur if the latter approach was used. Plotting values of autofluorescence of single pixels allows a better representation of small focal changes in fundus autofluorescence that may occur in some retinal diseases, such as those that be observed in patients with Stargardt macular dystrophyfundus flavimaculatus. 
Although cSLO autofluorescent images provide qualitative information on the topographic changes of fundus autofluorescence, quantitative data appear to be important in several circumstances. Thus, quantitative data are needed when levels of background fundus autofluorescence are to be evaluated. Similarly, it may be useful in determining if a particular area has a low level of fundus autofluorescence with respect to background or if levels of autofluorescence in that area are normal within the context of high background fundus autofluorescence.

Supported by the Macular Disease Society, United Kingdom (NL), and the Foundation Fighting Blindness, United States.

1 Feeney L. Lipofuscin and melanin of human retinal pigment epithelium. Fluorescence, enzyme cytochemical, and ultrastructural studies. Invest Ophthalmol Vis $\mathrm{Sci}$ 1978;17:583-600.

2 Feeney-Burns L, Berman ER, Rothman H. Lipofuscin of human retinal pigment epithelium. Am f Ophthalmol 1980; 90:783-91.

3 Boulton M, McKechnie NM, Breda J, et al. The formation of autofluorescent granules in cultures human RPE. Invest Ophthalmol Vis Sci 1989;30:82-9.

4 Feeney-Burns L, Eldred GE. The fate of the phagosome: conversion to "age pigment" and impact in human retinal pigment epithelium. Trans Ophthalmol Soc UK 1984;103: pigment

5 Katz ML, Drea C, Eldred G, et al. Influence of early photoreceptor degeneration on lipofuscin in the retinal pigphotoreceptor degeneration on lipofuscin in the

ment epithelium. Exp Eye Res 1986;43:561-73.
6 Young RW. Visual cells and the concept of renewal. Invest Ophthalmol 1976;15:700.

7 Wing GL, Blanchard GC, Weiter JJ. The topography and age relationship of lipofuscin concentration in the retinal pigment epithelium. Invest Ophthalmol Vis Sci 1978;17: 601-7.

8 Feeney-Burns L, Hilderbrand ES, Eldridge S. Aging human RPE: morphometric analysis of macular, equatorial, and peripheral cells. Invest Ophthalmol Vis Sci 1984;25:195-200.

9 Weiter JJ, Delori FC, Wing GL, et al. Retinal pigment epithelial lipofuscin and melanin and choroidal melanin in human eyes. Invest Ophthalmol Vis Sci 1986;27:145-52.

10 Weingeist TA, Kobrin JL, Watzke RC. Histopathology of Best's macular dystrophy. Arch Ophthalmol 1982;100: 1108-14.
11 Frangieh GT, Green WR, Fine SL. A histopathologic study of Best's macular dystrophy. Arch Ophthalmol 1982;100: 1115-21.

12 Eagle RC, Lucier AC, Bernardino VB, et al. Retinal pigment epithelial abnormalities in fundus flavimaculatus. A light and electron microscopic study. Ophthalmology 1980;87: 1189-200.

13 Birnbach CD, Järveläinen $M$, Possin DE, et al. Histopathology and immunocytochemistry of the neurosensory retina in fundus flavimaculatus. Ophthalmology 1994;101:121119.

14 Lopez PF, Maumenee IH, de la Cruz Z, et al. Autosomaldominant fundus flavimaculatus. Clinicopathologic correlation. Ophthalmology 1990;97:798-809.

15 Steinmetz RL, Garner A, Maguire JI, et al. Histopathology of incipient fundus flavimaculatus. Ophthalmology 1991;98: 953-6.

16 Dorey K, Staurenghi G, Delori FC. Lipofuscin in aged and ARMD eyes. In: Hollyfield JG, et al, eds. Retinal degeneration. New York: Plenum Press, 1993:3-14.

17 Dorey CK, Wu G, Ebenstein D, et al. Cell loss in the aging retina. Relationship to lipofuscin accumulation and macular degeneration. Invest Ophthalmol Vis Sci 1989;30: 1691-9.

18 Patrinely JR, Lewis RA, Font RL. Foveomacular vitelliform dystrophy, adult type. A clinicopathologic study including electron microscopic observations. Ophthalmology 1985;92: 1721-18.

19 Aguirre GD, Laties A. Pigment epithelial dystrophy in the dog. Exp Eye Res 1976;23:247-56.

20 Delori FC. Spectrophotometer for noninvasive measurements of intrinsic fluorescence and reflectance of the ocular fundus. Appl Optics 1994;33:7439-52.

21 Delori FC, Dorey CK, Staurenghi G, et al. In vivo fluorescence of the ocular fundus exhibits retinal pigment epithelium lipofuscin characteristics. Invest Ophthalmol Vis Sci 1995;36:718-29.

22 Delori FC, Staurenghi G, Arend O, et al. In vivo measurement of lipofuscin in Stargardt's disease-fundus flavimaculatus. Invest Ophthalmol Vis Sci 1995;36:2327-31.

23 Von Rückmann A, Fitzke FW, Bird AC. Distribution of fundus autofluorescence with a scanning laser ophthalmoscope. Br f Ophthalmol 1995;79:407-12.

24 Von Rückmann A, Fitzke FW, Bird AC. In vivo fundus autofluorescence in macular dystrophies. Arch Ophthalmol 1997;115:609-15.

25 Von Rückmann A, Fitzke FW, Bird AC. Fundus autofluorescence in age-related macular disease with a laser scanning ophthalmoscope. Invest Ophthalmol Vis Sci 1997; 38:478-86.

26 Wade AR, Fitzke FW. A fast, robust pattern recognition system for low light level image recognition and its application to retinal imaging. Optic Express 1998;3:190-7. 